\title{
NEW PATHOGENIC MUTATIONS ON GLDC GENE LEADING TO CLASSIC NONKETOTIC HYPERGLYCINEMIA
}

\author{
Teresa Almeida 1,2, Andreia Forno ${ }^{3,2}$, Luís Rodrigues ${ }^{1}$, Rita Jotta², Ana Luísa Rodrigues², Patrícia Janeiro², \\ Sandra Mexia ${ }^{4}$, Anabela Guerra ${ }^{4}$, José Matono${ }^{1}$, Isabel Tavares Almeida ${ }^{5}$, Ana Gaspar². \\ ${ }^{1}$ Pediatric Unit, Department of Women's and Child's Health, Hospital do Espírito Santo de Évora, EPE, \\ Évora, Portugal; \\ ${ }^{2}$ Reference Center for Metabolic Diseases, Pediatric Department, Hospital de Santa Maria, Centro \\ Hospitalar Universitário Lisboa Norte (CHULN), Lisboa, Portugal; \\ ${ }^{3}$ Department of Pediatrics, Hospital Central do Funchal, Madeira, Portugal; \\ ${ }^{4}$ Reference Center for Metabolic Diseases Dietetic and Nutrition Department, Hospital de Santa Maria, \\ Centro Hospitalar Universitário Lisboa Norte (CHULN), Lisboa, Portugal; \\ ${ }^{5}$ Met\&Ge, iMed.ULisboa, Faculdade de Farmácia, ULisboa, Lisbon, Portugal.
}

\section{KEYWORDS}

GLDC Gene, Nonketotic hyperglycinemia, mutations.

\section{ARTICLE HISTORY}

Received 11 January 2021

Accepted 2 February 2021
Nonketotic hyperglycinemia (NKH) is a rare autosomal recessive inborn error of metabolism due to deficient activity of glycine cleavage enzyme system. ${ }^{1}$ Although clinical presentation may be different according to the type of $\mathrm{NKH}$, frequent neonatal hiccups associated with altered state of consciousness and myoclonic seizures must raise high suspicion towards this rare disease leading to prompt diagnosis by a directed investigation. ${ }^{2}$ Differential diagnosis of a neonate presenting with encephalopathy without obvious metabolic derangement includes $\mathrm{NKH}$, urea cycle defects, maple syrup urine disease, organic acidurias, pyridoxine-dependent encephalopathy, and peroxisomal disorders. ${ }^{1,2}$

We report a first child of a non-consanguineous young couple, with an uneventful pregnancy and term dystocic hospital delivery. Due to feeding refusal by 16 hours of life, he was admitted to the neonatal intensive care unit and started on antibiotics. Along the third and fourth days of life, his clinical status progressively deteriorated and physical examination showed axial hypotonia with mild peripheral spasticity, persistent hiccups, myoclonic seizures, and hyperexcitability. Progressive lethargy led to coma and respiratory depression by the fifth day of life with the need for invasive mechanic ventilation for 22 days. Transcranial ultrasound and cerebral magnetic resonance Im aging performed on the fourth and fifth day of life both showed hypoplastic corpus callosum. Laboratory evaluation including complete blood count with differential, infection screen, kidney and liver function, blood glucose and electrolytes, uric acid, ammonia, lactate, and urinary organic acids chromatography were normal. Plasma,

Address for Correspondance: Teresa L. Almeida, Department of Pediatrics, Hospital do Espírito Santo de Évora, EPE, Évora, Portugal.

Email: teresaloualmeida@gmail.com

C2021 Pediatric Oncall urinary, and cerebrospinal fluid (CSF) glycine levels were elevated [1727.2 $\mu \mathrm{M}$ (Normal range: 223.8 -

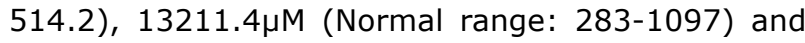
$321.6 \mu \mathrm{M}$ (Normal range 3.7-7.6) respectively]. Glycine ratio between CSF and plasma was also elevated [0.19 (Normal range: <0,04)]. Electroencephalogram revealed a burst-suppression intermittent pattern consistent with NKH encephalopathy. Molecular diagnosis identified two undescribed heterozygous variants (c.1406G >A and c.2690G>C) on the GLDC gene and in silico predictions suggested pathogenicity. He started treatment on the seventh day of life with sodium benzoate, dextromethorphan, calcium folinate, pyridoxine, clonazepam, and phenobarbital. No clinical improvement was attained despite normal glycine values by the twelfth day of life. Sodium benzoate as high as $600 \mathrm{mg} / \mathrm{kg} /$ day was needed to normalize glycine levels which led to a severe generalized eczematous pruritic cutaneous rash. After more than one year of therapy optimization and multidisciplinary follow-up, severe neurodevelopmental impairment and incomplete seizure control persisted.

Mutations on GLDC and AMT genes are associated with $\mathrm{NKH}$ and this case presents two undescribed variants of the GLDC gene clinically associated with severe classic $\mathrm{NKH}$ with neonatal metabolic encephalopathy. ${ }^{2,3}$ Despite optimized treatment with normalization of CSF glycine levels, our patient's severe neurodevelopmental outcome remained unchanged is reserved, as described in the literature. ${ }^{4}$ To date, there are no effective treatment options for NKH and current treatment can't improve the clinical status, even with normal plasma and CSF glycine levels. ${ }^{1}$ Side effects such as the observed cutaneous rash must be minimized, despite the possible elevation of glycine levels to maintain patient's quality of life. A multidisciplinary approach is essential when dealing with these complex patients including neonatology, pediatric neurology, metabolic, genetic, and palliative care specialists. ${ }^{1}$ 


\section{Compliance with Ethical Standards}

Funding: None

Conflict of Interest: None

\section{References}

1. Bharti K, Bajaj N. Nonketotic hyperglycinemia. Perinatology. 2020;18(1):28-30.

2. Iqbal M, Prasad M, Mordekar SR. Nonketotic hyperglycinemia case series. J Pediatr Neurosci. 2015;10(4):355-8.
3. Coughlin $C R$, Swanson MA, Kronquist $K$, Acquaviva $C$, Hutchin $\mathrm{T}$, Rodríguez-Pombo $\mathrm{P}$, et al. The genetic basis of classic nonketotic hyperglycinemia due to mutations in GLDC and AMT. Genet Med. 2017;19(1):104-11.

4. Bjoraker KJ, Swanson MA, Coughlin CR, Christodoulou J, Tan ES, Fergeson M, et al. Neurodevelopmental Outcome and Treatment Efficacy of Benzoate and Dextromethorphan in Siblings with Attenuated Nonketotic Hyperglycinemia. J Pediatr. 2016;170:234-9. 